\title{
Exame citopatológico do colo do útero: fatores associados a não realização em ESF ${ }^{1}$

\author{
Cytopathologic examination of uterine cervical: factors associated with not \\ performing in FHS
}

\section{Examinación de la citología cervicouterina: factores asociados no la realización en ESF}

\author{
J oão Felício Rodrigues Neto', Maria Fernanda Santos Figueiredo", Leila das Graças Siqueira"I
}

\section{RESUMO}

A principal estratégia para deteç̧ão precoce do câncer do colo do útero é o exame citopatológico. Este estudo tem o objetivo de identificar os principais fatores culturais, sociais, econômicos, aspectos sexuais e reprodutivos e os motivos para a não realização do exame citopatológico em mulheres; e verificar uma possível associação destas variáveis com seu conhecimento sobre a finalidade desse exame. Trata-se de um estudo transversal, com a seleção de 45 mulheres cadastradas em três equipes da Estratégia Saúde da Família de Montes Claros (MG), no período de setembro a dezembro de 2006 . Observou-se que $71,1 \%$ das pesquisadas têm mais de 50 anos, $28,9 \%$ são analfabetas e $73,3 \%$ tem renda mensal entre $\mathrm{R} \$ 300,00$ a $\mathrm{R} \$ 600,00$. O conhecimento sobre a finalidade do exame associou-se a cor não branca $(p=0,019)$; conceito correto sobre a finalidade do exame $(p=0,001)$ não ter companheiro $(p=0,030)$ e trabalhar $(p=0,001)$. O principal motivo para a não realização do exame $(53,3 \%)$ foi o fato de não estarem doentes. Assim, a maior parte das mulheres que não realizam o exame possui idade avançada; com menor escolaridade e menor nível socioeconômico. O conhecimento da finalidade do exame está associado à cor, ao conceito correto, ao estado conjugal e a ocupação.

Palavras chave: Neoplasias do colo do útero; Esfregaço vaginal; Programa Saúde da Família.

\footnotetext{
ABSTRACT

The main strategy for precocious detention of the cervical cancer is the cytopathologic examination. This objective study to identify the main cultural, social, economic factors, sexual and reproductive aspects and reasons for not the accomplishment of the cytopathologic examination in women; to verify a possible association of these variables with its knowledge on the purpose of this examination. This one is about a transversal study, whith the selection of 45 women registered in cadastre in
}

three teams of the Family Health Strategy of Montes Claros (MG), in the period from September to December of 2006. It was realized the descriptive analysis and statistics, whose considered significance was $p<0,05$. $71,1 \%$ of the searched from 50 years old one, $28,9 \%$ are illiterate and $73,3 \%$ have monthly income between $R \$ 300,00$ the $R \$ 600,00$. The knowledge on the purpose of the examination associated it not white color $(p=0,019)$; correct concept on the purpose of the examination $(p=0,001)$ not to have spouse $(p=0,030)$ and to work $(p=0,001)$. The main reason for the accomplishment of the examination $(53,3 \%)$ was not the fact not to be sick. Thus, most of the women who do not realize the examination have advanced age; with low level of education and minor socio-economic level.

Key words: Uterine cervical neoplasms; Vaginal smears; Family Health Program.

\section{RESUMEN}

La estrategia principal para la detención precocious del cáncer del cuello uterino es citología cervicouterina. Este estudio objetivo para identificar factores culturales, sociales, económicos principales, sexuales y reproductivos y las razones no de la realización de la examinación de la citología; para verificar una asociación posible de estos variables con su conocimiento en el propósito de esta examinación. Es un estudio transversal, com la

\footnotetext{
${ }^{1}$ Artigo desenvolvido a partir da Monografia da autora Maria Fernanda Santos Figueiredo

Médico. Professor Doutor do Departamento de Clínica Médica da Universidade Estadual de Montes Claros, Montes Claros, MG, Brasil. Email: joao.felicio@unimontes.br.

"Enfermeira. Professora do Departamento de Enfermagem das Faculdades Unidas do Norte de Minas e Faculdades Integradas Pitágoras. Montes Claros, MG, Brasil. Email: nanda_sanfig@yahoo.com.br.

III Enfermeira. Professora Mestre do Departamento de Enfermagem da Universidade Estadual de Montes Claros; das Faculdades Unidas do Norte de Minas e Faculdades Integradas Pitágoras. Montes Claros, MG, Brasil. Email: siqle@ig.com.br.
} 
Rodrigues Neto JF, Figueiredo MFS, Siqueira LG. Exame citopatológico do colo do útero: fatores associados a não realização em ESF. Rev. Eletr. Enf. [Internet]. 2008;10(3):610-21. Available from: http://www.fen.ufg.br/revista/v10/n3/v10n3a07.htm

selección de 45 mujeres registradas en cadastre en tres equipos del Programa de la Salud de la Familia em Montes Claros (MG) em el período de setiembre a diciembre de 2006. Hizo el análisis descriptivo y la estadística, que consideraban significación era $\mathrm{p}<0,05$. $71,1 \%$ tienen 50 años más $28,9 \%$ son analfabetos y $73,3 \%$ tienen renta mensual entre $\mathrm{R} \$ 300,00$ el $\mathrm{R} \$ 600,00$. El conocimiento en el propósito de la examinación la asoció el color no blanco $(p=0,019)$; concepto correcto en el propósito de

\section{NTRODUÇÃO}

O câncer tem sido responsável por seis milhões de mortes por ano, correspondendo a $12 \%$ das mortes mundiais. Estima-se a ocorrência de mais de dez milhões de novos casos de câncer por ano, sendo que para o ano de 2020, espera-se que haja mais de 15 milhões de casos novos ${ }^{(1-2)}$.

O câncer do colo do útero (CCU) ocupa o 2 o lugar na incidência mundial de neoplasias, estando atrás, apenas do câncer de mama(3). Por ano, no mundo, ocorrem cerca de $235 \mathrm{mil}$ mortes devido ao CCU, sendo 218 mil em países considerados pobres ou emergentes ${ }^{(4)}$, onde ocorre quase $60 \%$ dos casos novos de câncer ${ }^{(3)}$, ocupando um lugar de destaque nas taxas de morbimortalidade entre a população feminina ${ }^{(5)}$. Ressalta-se que na América Latina se encontram as mais altas taxas de incidência desta doença, representando uma das causas de óbito mais freqüente entre as mulheres ${ }^{(6)}$. No Brasil, são esperados 18.680 casos novos deste câncer para o ano de 2008, constituindo um risco estimado de 19 casos a cada 100.000 mulheres, sendo que destes, 4.720 ocorrerão em mulheres da região Nordeste. Não considerando os carcinomas de pele não melanoma, o CCU é o mais incidente na Região Norte $(22 / 100.000)$. Já, nas regiões Sul (24/100.000), Centro-Oeste $(19 / 100.000)$ e Nordeste (18/100.000) ocupa a segunda posição e no Sudeste $(18 / 100.000)$ a quarta posição ${ }^{(3)}$, estando assim associado a regiões brasileiras de baixo nível socioeconômico ${ }^{(7-8)}$.

Ressalta-se que apesar desse tipo de câncer apresentar aspectos epidemiológicos, etiológicos e evolutivos bem conhecidos, o que permite sua detecção em estágio pré-neoplásico ou inicial ${ }^{(2)}$, constata-se ainda a dura realidade, la examinación $(p=0,001)$ de no tener esposo $(p=0,030)$ y de no trabajar $(p=0,001)$. La razón principal de la realización de la examinación no era el hecho a no ser enfermo (53,3\%). Así, la mayor parte de las mujeres que no llevan através de la examinación han edad avanzada; con poco escolaridade y menor nivel sócioeconómico.

Palabras clave: Neoplasias del cuello uterino; Frotis vaginal; Programa Salud de la Família.

onde a permanência de elevadas taxas de sua incidência e de sua mortalidade deve-se ao perfil epidemiológico que essa patologia adquire nos países, além da freqüência dos fatores de risco, e, principalmente do grau de implementação de ações efetivas de curtos e longos prazos, tanto no plano técnico, no diagnóstico precoce e tratamento das lesões detectadas, quanto nos planos educacional, social e político-econômico(9-11). Apesar do exame citopatológico do colo do útero (ECCU) ter sido comprovado como uma técnica efetiva e eficiente em diminuir as taxas de morbimortalidade ${ }^{(3,5,12)}$, a sua cobertura ainda é insuficiente, devido a fatores socioeconômicos ${ }^{(4,10,13)}$, culturais $^{(4,8,12)}$, sexuais e reprodutivos ${ }^{(4,10,12,14)}$, além dos fatores relativos à assistência em saúde ${ }^{(5-6,9)}$. Bem como, ressalta-se também que as crenças, sentimentos e atitudes em relação ao $\mathrm{CCU}$ e ao ECCU também influenciam como fatores na adesão da mulher ao exame citopatológico $^{(2,6,8,14)}$. Destaca-se que não existe consenso em relação à idade em que não é mais necessária a realização do $\mathrm{ECCU}^{(7)}$.

Este trabalho se justifica pela escassez de estudos realizados no Brasil sobre os fatores associados à não realização do $\mathrm{ECCU}^{(4)}$ e que avaliem os fatores que dificultam ou favorecem sua adesão ao exame, visto que a realização desse exame é a principal estratégia utilizada para detecção precoce do $\mathrm{CCU}^{(3)}$. Estudos com essa temática realizados no Norte de Minas Gerais, ainda são mais escassos, sendo de fundamental relevância, por se tratar de uma região de baixos indicadores socioeconômicos e culturais e pelo fato de fazer parte da região geoeconômica do Nordeste brasileiro, que ocupa a $2^{a}$ posição na incidência do $\mathrm{CCU}^{(6)}$. 
Rodrigues Neto JF, Figueiredo MFS, Siqueira LG. Exame citopatológico do colo do útero: fatores associados a não realização em ESF. Rev. Eletr. Enf. [Internet]. 2008;10(3):610-21. Available from: http://www.fen.ufg. br/revista/v10/n3/v10n3a07.htm

Destaca-se ainda a importância desse estudo ter sido realizado em equipes da Estratégia Saúde da Família (ESF), já que grande parte dos estudos sobre o CCU e a realização do seu ECCU são realizado em ambulatórios $^{(4,7,8,11-12)}$ e hospitais ${ }^{(9)}$ e a ESF, que vem sendo implementada nos últimos anos, constitui importante palco com ferramentas imprescindíveis para aumentar a cobertura de prevenção dessa doença e atingir especificamente os grupos de maior risco ${ }^{(9)}$.

O objetivo deste estudo foi identificar os principais fatores culturais, sociais, econômicos, aspectos sexuais, reprodutivos e os motivos para a não realização do ECCU em mulheres cadastradas na ESF; e verificar uma possível associação destas variáveis com seu conhecimento sobre a finalidade desse exame.

\section{MÉTODOS}

Este trabalho foi aprovado pelo Comitê de Ética e Pesquisa (CEP) da Universidade Estadual de Montes Claros, conforme parecer número $419 / 06$.

O estudo realizado foi do tipo transversal, de abordagem quantitativa e de caráter descritivo. A pesquisa foi realizada na cidade de Montes Claros, norte de Minas Gerais (MG), que apresenta 330.000 habitantes e possui $46,7 \%$ de área de cobertura da ESF, possuindo 48 equipes implantadas, sendo 43 na zona urbana e cinco na zona rural. Para este estudo, foram selecionadas três equipes de ESF da zona urbana: Jardim Palmeiras I, Jardim Palmeiras II e Delfino Magalhães, que constituem a área denominada Grande Delfino, importante setor da cidade, que possui 8216 mil habitantes, sendo que 2964 são mulheres com 20 anos ou mais.

A amostragem deste trabalho foi constituída por mulheres, obedecendo aos seguintes critérios de inclusão: idade a partir de 20 anos, de acordo à classificação etária utilizada nos registros de consolidação anual das famílias cadastradas por área (Relatório A2) do Sistema de Informação de Atenção Básica $(\mathrm{SIAB})^{(15)}$; cadastro nas equipes selecionadas da ESF, a partir dos dados do $\mathrm{SIAB}^{(15)}$; nunca ter realizado o ECCU ou ter o realizado a mais de três anos, conforme registros dos Agentes
Comunitários de Saúde (ACS) das respectivas áreas. Os critérios de exclusão estabelecidos foram: mulheres cadastradas que realizaram o ECCU nos últimos três anos e não aceitação em participar do estudo.

O instrumento utilizado para a coleta dos dados foi o formulário que contemplava variáveis sociais e econômicas: cor, escolaridade, estado conjugal, idade, ocupação principal, religião e renda mensal familiar; sexuais e reprodutivas: idade de início das atividades sexuais, número total de parceiros sexuais, número total de gestações e uso de contraceptivo; e culturais: conhecimento sobre - ECCU e motivos para a sua não realização. Para verificar o conhecimento sobre a finalidade do ECCU, a seguinte questão foi formulada: "Qual a finalidade do exame citopatológico do colo do útero em sua opinião?". Cinco opções de respostas foram dadas às pesquisadas: não sei; para prevenir ou detectar precocemente o câncer do colo do útero; detectar doenças sexualmente transmissíveis ou doenças ginecológicas; detectar alterações no útero; ou detectar outras doenças. A opção considerada correta foi: prevenir ou detectar precocemente o CCU.

A coleta de dados foi realizada pela pesquisadora, que após a identificação das mulheres que constituíam a amostragem, se dirigia até o local de residência das mesmas, onde aplicava os formulários, após o aceito da entrevistada em participar da pesquisa e leitura do Termo de Consentimento Livre e Esclarecido.

Após levantamento dos dados durante setembro e outubro de 2006, os mesmos foram compilados e estruturados em um banco de dados e posteriormente, submetidos à análise descritiva e estatística dos dados. Utilizou-se o programa estatístico Statístical Pocckage for the Social Sciences (SPSS) for Windows versão 15.0, para a realização do teste de Quiquadrado $\left(\chi^{2}\right)$, a fim de verificar associação entre as variáveis independentes: cor, conceito sobre a finalidade do exame, consulta ao serviço de saúde, escolaridade, estado conjugal, idade, religião, renda familiar, ocupação, número de gestações e número de filhos - e a variável resposta - conhecimento sobre a finalidade do exame citopatológico do colo do 
Rodrigues Neto JF, Figueiredo MFS, Siqueira LG. Exame citopatológico do colo do útero: fatores associados a não realização em ESF. Rev. Eletr. Enf. [Internet]. 2008;10(3):610-21. Available from: http://www.fen.ufg.br/revista/v10/n3/v10n3a07.htm

útero. Para o teste de significância estatística foi considerado $\mathrm{p}<0,05$.

\section{RESULTADOS}

O presente estudo selecionou 2964 mulheres de acordo com os critérios de inclusão estabelecidos, sendo que $45(1,5 \%)$ mulheres constituíram a amostragem desse trabalho.

Quanto ao perfil socioeconômico das 45 pesquisadas, $32(71,1 \%)$ mulheres tinham mais de 50 anos; $14(31,1 \%)$ relataram serem casadas; $13(28,9 \%)$ analfabetas; e $33(73,3 \%)$ referiram que a renda mensal de sua família estava entre $R \$ 300,00$ a $R \$ 600,00$. Foi verificado que $35(77,8 \%)$ mulheres não trabalhavam atualmente, sendo que destas 32 $(91,4 \%)$ referiram como a ocupação principal o cuidado com o "Lar". Tabela 1.

Tabela 1: Perfil socioeconômico das mulheres que não realizam o exame citopatológico do colo do útero da área de abrangência das Estratégias Saúde da Família Delfino Magalhães e Jardim Palmeiras I e II, Montes Claros, Outubro-2006

\begin{tabular}{|c|c|c|}
\hline Variáveis & No & $\%$ \\
\hline \multicolumn{3}{|l|}{ Cor } \\
\hline Branca & 19 & 42,2 \\
\hline Parda/mulata & 20 & 44,5 \\
\hline Negra & 06 & 13,3 \\
\hline Amarela & 00 & 0,0 \\
\hline Outros & 00 & 0,0 \\
\hline \multicolumn{3}{|l|}{ Escolaridade } \\
\hline Analfabeto (nunca estudou) & 13 & 28,9 \\
\hline Ensino fundamental incompleto (até oito anos de estudo) & 16 & 35,6 \\
\hline Ensino fundamental completo (oito anos de estudo) & 06 & 13,3 \\
\hline Ensino médio incompleto (até 11 anos de estudo) & 02 & 4,4 \\
\hline Ensino médio completo ( 11 anos de estudo) & 05 & 11,1 \\
\hline Ensino superior incompleto & 02 & 4,4 \\
\hline Ensino superior completo & 01 & 2,2 \\
\hline \multicolumn{3}{|l|}{ Estado Conjugal } \\
\hline Solteiro & 10 & 22,2 \\
\hline Casado & 14 & 31,1 \\
\hline União Consensual & 05 & 11,1 \\
\hline Separado & 03 & 6,7 \\
\hline Divorciado & 00 & 0,0 \\
\hline Viúvo & 13 & 28,9 \\
\hline \multicolumn{3}{|l|}{ I dade } \\
\hline 20 a 29 anos & 03 & 6,7 \\
\hline 30 a 39 anos & 05 & 11,1 \\
\hline 40 a 49 anos & 05 & 11,1 \\
\hline 50 anos ou mais & 32 & 71,1 \\
\hline \multicolumn{3}{|l|}{ Ocupação Principal } \\
\hline Trabalha por conta própria & 06 & 13,3 \\
\hline Empregado & 04 & 8,9 \\
\hline Não trabalha & 35 & 77,8 \\
\hline \multicolumn{3}{|l|}{ Religião } \\
\hline Católica & 33 & 73,3 \\
\hline Evangélica & 12 & 26,7 \\
\hline Outras & 00 & 0,0 \\
\hline Não tem religião & 00 & 0,0 \\
\hline \multicolumn{3}{|l|}{ Renda Mensal Familiar } \\
\hline Até $\mathrm{R} \$ 300,00$ & 05 & 11,1 \\
\hline De $R \$ 301,00$ a $R \$ 600,00$ & 33 & 73,3 \\
\hline De $R \$ 601,00$ a $R \$ 1200,00$ & 07 & 15,6 \\
\hline De $R \$ 1201,00$ a $R \$ 2400,00$ & 00 & 0,0 \\
\hline De $R \$ 2401,00$ a $R \$ 4800,00$ & 00 & 0,0 \\
\hline Mais de $\mathrm{R} \$ 4800,00$ & 00 & 0,0 \\
\hline
\end{tabular}

Fonte: Dados coletados, ESF Delfino Magalhães e Jardim Palmeiras I e II, 2006. 
Rodrigues Neto JF, Figueiredo MFS, Siqueira LG. Exame citopatológico do colo do útero: fatores associados a não realização em ESF. Rev. Eletr. Enf. [Internet]. 2008;10(3):610-21. Available from: http://www.fen.ufg.br/revista/v10/n3/v10n3a07.htm

Quanto aos aspectos sexuais e reprodutivos das entrevistadas, verificou-se que $16(35,6 \%)$ tiveram o início da atividade sexual com 20 anos ou mais; $24(53,3 \%)$ dessas mulheres tiveram apenas um parceiro sexual, desde o início da sua atividade sexual; 17 $(37,8 \%)$ referiram ter tido sete ou mais gestações. Tabela 2.

Tabela 2: Perfil sexual e reprodutivo das mulheres que não realizam o exame citopatológico do colo do útero de abrangência das Estratégias Saúde da Família Delfino Magalhães e Jardim Palmeiras I e II, Montes Claros, Outubro-2006

\begin{tabular}{|c|c|c|}
\hline Variáveis & № & $\%$ \\
\hline \multicolumn{3}{|l|}{ I dade de início das atividades sexuais } \\
\hline Nunca tiveram relação sexual & 06 & 13,3 \\
\hline Até 15 anos & 06 & 13,3 \\
\hline $16-17$ anos & 06 & 13,3 \\
\hline 18-19 anos & 11 & 24,5 \\
\hline 20 anos ou mais & 16 & 35,6 \\
\hline \multicolumn{3}{|l|}{ Número total de parceiros sexuais } \\
\hline Nenhum & 06 & 13,3 \\
\hline 1 & 24 & 53,3 \\
\hline 2 a 6 & 13 & 28,9 \\
\hline 7 ou mais & 02 & 4,4 \\
\hline \multicolumn{3}{|l|}{ Número total de gestações } \\
\hline Nenhuma & 06 & 13,3 \\
\hline 1 & 04 & 8,9 \\
\hline 2 a 6 & 18 & 40,0 \\
\hline 7 ou mais & 17 & 37,8 \\
\hline \multicolumn{3}{|l|}{ Número total de filhos } \\
\hline Nenhum & 06 & 13,3 \\
\hline 1 & 04 & 8,9 \\
\hline 2 a 6 & 19 & 42,2 \\
\hline 7 ou mais & 16 & 35,6 \\
\hline \multicolumn{3}{|l|}{ Uso de contraceptivo } \\
\hline Utiliza algum tipo de contraceptivo & 02 & 4,4 \\
\hline Não utiliza contraceptivo & 43 & 95,6 \\
\hline
\end{tabular}

Fonte: Dados coletados, ESF Delfino Magalhães e Jardim Palmeiras I e II, 2006.

Entre as $32(71,1 \%)$ mulheres de 50 anos ou mais que não realizam o ECCU, verificou-se que, quanto ao seu perfil socioeconômico, 26 $(81,2 \%)$ possuíam até oito anos de estudo; 27 $(84,4 \%)$ possuíam renda familiar mensal de até $\mathrm{R} \$ 600,00$ e 28 (87,5\%) não trabalhavam. Quantos aos aspectos sexuais e reprodutivos, $21(80,8 \%)$ iniciaram a vida sexual com 18 anos ou mais, excetuando-se aquelas que nunca tiveram relação sexual, e $17(53,1 \%)$ tiveram quatro ou mais filhos. A não realização do exame citopatológico em mulheres acima de 50 anos esteve associada as variáveis: escolaridade $\quad\left(\chi^{2} \quad=11,23234 ; \quad \mathrm{p}=0,000\right)$; ocupação principal $\left(\chi^{2}=4,267041 ; 0,038\right)$ e idade de início da atividade sexual $\left(\chi^{2}\right.$ $=4,7433319 ; p=0,029$ ). Tabela 3 . 
Rodrigues Neto JF, Figueiredo MFS, Siqueira LG. Exame citopatológico do colo do útero: fatores associados a não realização em ESF. Rev. Eletr. Enf. [Internet]. 2008;10(3):610-21. Available from: http://www.fen.ufg.br/revista/v10/n3/v10n3a07.htm

Tabela 3: Perfil socioeconômico, sexual e reprodutivo e cultural das mulheres a partir de 50 anos que não realizam o exame citopatológico do colo do útero da área de abrangência das Estratégias Saúde da Família Delfino Magalhães e Jardim Palmeiras I e II, Montes Claros, Outubro-2006

\begin{tabular}{|c|c|c|c|c|c|c|}
\hline \multirow{2}{*}{\begin{tabular}{l}
\multicolumn{1}{c}{ Variáveis } \\
Características socioeconômicas \\
Cor
\end{tabular}} & \multicolumn{2}{|c|}{ De 18 a 49 anos } & \multicolumn{2}{|c|}{50 anos ou mais } & \multirow[t]{2}{*}{$\chi^{2}$} & \multirow[t]{2}{*}{$\mathbf{P}$} \\
\hline & $\mathbf{N}^{\circ}$ & $\%$ & № & $\%$ & & \\
\hline Branca & 06 & 46,2 & 13 & 40,6 & 0,0000547 & 0,994 \\
\hline Não Branca & 07 & 53,8 & 19 & 59,4 & & \\
\hline \multicolumn{7}{|l|}{ Escolaridade } \\
\hline Até oito anos de estudo & 03 & 23,1 & 26 & 81,2 & 11,23234 & 0,000 \\
\hline Oito anos ou mais de estudo & 10 & 76,9 & 06 & 18,8 & & \\
\hline \multicolumn{7}{|l|}{ Estado Conjugal } \\
\hline Vive com um companheiro & 08 & 61,5 & 11 & 34,4 & 1,7934504 & 0,180 \\
\hline Não vive com um companheiro & 05 & 38,5 & 21 & 65,6 & & \\
\hline \multicolumn{7}{|l|}{ Ocupação Principal } \\
\hline Trabalha & 06 & 46,2 & 04 & 12,5 & 4,267041 & 0,038 \\
\hline Não trabalha & 07 & 53,8 & 28 & 87,5 & & \\
\hline \multicolumn{7}{|l|}{ Religião } \\
\hline Presença de prática religiosa & 13 & 100 & 32 & 100 & - & - \\
\hline Ausência de prática religiosa & 00 & 00 & 00 & 00 & & \\
\hline \multicolumn{7}{|l|}{ Renda Mensal Familiar } \\
\hline Até $\mathrm{R} \$ 600,00$ & 11 & 84,6 & 27 & 84,4 & 0,00000 & 1,000 \\
\hline $\mathrm{R} \$ 600,00$ ou mais & 02 & 15,4 & 05 & 15,6 & & \\
\hline \multicolumn{7}{|c|}{$\begin{array}{l}\text { Características sexuais e reprodutivas } \\
\text { I nicio das atividades sexuais* }\end{array}$} \\
\hline Até 17 anos & 07 & 53,8 & 05 & 19,2 & 4,7433319 & 0,029 \\
\hline 18 anos ou mais & 06 & 46,2 & 21 & 80,8 & & \\
\hline \multicolumn{7}{|l|}{ Número de parceiros sexuais* } \\
\hline 1 parceiro sexual & 08 & 61,5 & 16 & 61,5 & 0,00000 & 1,000 \\
\hline 2 parceiros sexuais ou mais & 05 & 38,5 & 10 & 38,5 & & \\
\hline \multicolumn{7}{|l|}{ Número total de gestações } \\
\hline De nenhuma até três gestações & 08 & 61,5 & 12 & 37,5 & 1,299429 & 0,254 \\
\hline Quatro gestações ou mais & 05 & 38,5 & 20 & 62,5 & & \\
\hline \multicolumn{7}{|l|}{ Número total de filhos } \\
\hline De nenhum até três filhos & 08 & 61,8 & 15 & 46,9 & 0,3168766 & 0,573 \\
\hline Quatro filhos ou mais & 05 & 38,5 & 17 & 53,1 & & \\
\hline
\end{tabular}

Fonte: Dados coletados, ESF Delfino Magalhães e Jardim Palmeiras I e II,2006.

* Foram excluídas seis mulheres que nunca tiveram relação sexual

Outro aspecto pesquisado neste estudo, refere-se ao conhecimento das mulheres sobre - ECCU e a sua finalidade. Os resultados demonstraram que 42 (93,3\%) mulheres já ouviram falar sobre esse exame, entretanto, 11 $(26,2 \%)$ destas afirmaram não conhecer a finalidade do mesmo. Entre as 31 (68,9\%) mulheres que relataram ter conhecimento sobre a finalidade do exame, verificou-se que quanto ao perfil socioeconômico, 22 (70,9\%) possuíam 50 anos ou mais e apresentavam cor não branca; $26(83,9 \%)$ possuíam renda familiar de até $R \$ 600,00$. Já quanto aos aspectos sexuais e reprodutivos, observou-se que 17 (54,8\%) tiveram quatro gestações ou mais. A conceituação incorreta sobre a finalidade deste exame foi observada em $14(45,2 \%)$ das que diziam ter conhecimento sobre a mesma. Os resultados possibilitaram descrever que o conhecimento das mulheres sobre a finalidade do ECCU esteve associado aos seguintes fatores: $\operatorname{cor}\left(\chi^{2}=5,474454 ; p=0,019\right)$; conceituação correta sobre a finalidade do mesmo $\left(\chi^{2}=10,116036 ; p=0,001\right)$; estado conjugal $\left(\chi^{2}=4,678859 ; p=0,030\right)$ e ocupação principal $\left(\chi^{2}=10,3729838 ; p=0,001\right)$ (Tabela 4). Destaca-se que as principais fontes de conhecimento sobre o exame foram seus conhecidos e os meios de comunicação como TV, rádio, jornal ou revista, sendo estes citados respectivamente por $22(48,9 \%)$ e $13(28,9 \%)$ das entrevistadas. 
Rodrigues Neto JF, Figueiredo MFS, Siqueira LG. Exame citopatológico do colo do útero: fatores associados a não realização em ESF. Rev. Eletr. Enf. [Internet]. 2008;10(3):610-21. Available from: http://www. fen.ufg.br/revista/v10/n3/v10n3a07.htm

Tabela 4: Conhecimento das mulheres sobre a finalidade do exame citopatológico do colo do útero e as variáveis socioeconômicas, sexuais, reprodutivas e culturais, da área de abrangência das Estratégias Saúde da Família Delfino Magalhães e Jardim Palmeiras I e II, Montes Claros, Outubro-2006

\begin{tabular}{|c|c|c|c|c|c|c|}
\hline \multirow{3}{*}{ Variáveis } & \multicolumn{4}{|c|}{$\begin{array}{c}\text { Conhecimento sobre a finalidade do } \\
\text { exame }\end{array}$} & \multirow{3}{*}{$\chi^{2}$} & \multirow{3}{*}{$\mathbf{P}$} \\
\hline & \multicolumn{2}{|c|}{ Sim } & \multicolumn{2}{|c|}{ Não } & & \\
\hline & № & $\%$ & No & $\%$ & & \\
\hline \multicolumn{7}{|l|}{ Cor } \\
\hline Branca & 09 & 29,1 & 10 & 71,4 & 5,474454 & 0,019 \\
\hline Não Branca & 22 & 70,9 & 04 & 28,6 & & \\
\hline \multicolumn{7}{|l|}{$\begin{array}{l}\text { Conceito sobre a finalidade do } \\
\text { exame }\end{array}$} \\
\hline Correto & 17 & 54,8 & 00 & 0,0 & 10,116036 & 0,001 \\
\hline Incorreto & 14 & 45,2 & 14 & 100 & & \\
\hline \multicolumn{7}{|l|}{ Consulta ao serviço de saúde } \\
\hline $\operatorname{Sim}$ & 29 & 93,5 & 10 & 71,4 & 2,3937655 & 0,121 \\
\hline Não & 02 & 6,5 & 04 & 28,6 & & \\
\hline \multicolumn{7}{|l|}{ Escolaridade } \\
\hline Até oito anos de estudo & 19 & 61,3 & 10 & 71,4 & 0,1032956 & 0,747 \\
\hline Oito anos ou mais de estudo & 12 & 38,7 & 04 & 28,6 & & \\
\hline \multicolumn{7}{|l|}{ Estado Conjugal } \\
\hline Vive com Companheiro & 14 & 45,2 & 01 & 7,1 & 4,678859 & 0,030 \\
\hline Vive sem Companheiro & 17 & 54,8 & 13 & 92,9 & & \\
\hline \multicolumn{7}{|l|}{ I dade } \\
\hline $18-49$ & 09 & 29,1 & 04 & 28,6 & 0,00000 & 1,000 \\
\hline 50 ou + & 22 & 70,9 & 10 & 71,4 & & \\
\hline \multicolumn{7}{|l|}{ Religião } \\
\hline Presença de prática religiosa & 31 & 100 & 14 & 100 & $-*$ & - \\
\hline Ausência de prática religiosa & 00 & 0,0 & 00 & 0,0 & & \\
\hline \multicolumn{7}{|l|}{ Renda Familiar } \\
\hline Até $\mathrm{R} \$ 600,00$ & 26 & 83,9 & 10 & 71,4 & 0,3175403 & 0,573 \\
\hline$>\mathrm{R} \$ 600,00$ & 05 & 16,1 & 04 & 28,6 & & \\
\hline \multicolumn{7}{|l|}{ Ocupação } \\
\hline Trabalha & 24 & 77,4 & 03 & 21,4 & 10,3729838 & 0,001 \\
\hline Não Trabalha & 07 & 25,6 & 11 & 78,6 & & \\
\hline \multicolumn{7}{|l|}{ Número de Gestações } \\
\hline Até três gestações & 14 & 45,2 & 06 & 42,9 & 0,0207672 & 0,885 \\
\hline Quatro ou mais gestações & 17 & 54,8 & 08 & 57,1 & & \\
\hline \multicolumn{7}{|l|}{ Número de Filhos } \\
\hline Até três filhos & 16 & 51,6 & 07 & 50 & 0,0100408 & 0,920 \\
\hline Quatro ou mais & 15 & 48,4 & 07 & 50 & & \\
\hline
\end{tabular}

Fonte: Dados coletados, ESF Delfino Magalhães e Jardim Palmeiras I e II, 2006.

Além disso, verificou-se que os principais motivos relatados pelas entrevistadas para não realização do ECCU foram o fato de não estarem doente ou não apresentarem nenhuma sintomatologia, seguido pelo fato de terem vergonha ou sentirem constrangidas, perfazendo um total de $24(53,3 \%)$ e 15 $(33,3 \%)$ respectivamente. Entre as mulheres com idade acima de 50 anos, foram também esses os principais motivos referidos, totalizando respectivamente $21(65,6 \%)$ e 9 $(28,1 \%)$. Tabela 5 
Rodrigues Neto JF, Figueiredo MFS, Siqueira LG. Exame citopatológico do colo do útero: fatores associados a não realização em ESF. Rev. Eletr. Enf. [Internet]. 2008;10(3):610-21. Available from: http://www.fen.ufg.br/revista/v10/n3/v10n3a07.htm

Tabela 5. Motivos para a não realização do exame citopatológicodo colo do útero pelas mulheres da área de abrangência das Estratégias Saúde da Família Delfino Magalhães

e Jardim Palmeiras I e II, Montes Claros, Outubro-2006

\begin{tabular}{lccc}
\hline \multirow{2}{*}{ Motivos para não realização do exame citopatológico* } & \multicolumn{2}{c}{$\begin{array}{c}\text { Mulheres com mais } \\
\text { de 50 anos }\end{array}$} & \multicolumn{2}{c}{ Todas as mulheres } \\
\cline { 2 - 4 } & $\mathbf{N o}$ & \% & No \\
\hline Não está doente ou não apresenta nenhuma sintomatologia & 21 & 65,6 & 24 \\
Por vergonha ou constrangimento & 09 & 28,1 & 15 \\
O exame é doloroso & 05 & 15,6 & 08 \\
Por descuido & 01 & 3,1 & 05 \\
Tem medo do resultado do exame & 05 & 15,6 & 06 \\
Não sabia do exame ou não conhecia a sua finalidade & 03 & 9,4 & 04 \\
Não tem tempo disponível ou não pode faltar o trabalho & 00 & 0,0 & 03 \\
Ó médico ou enfermeiro não solicita a realização & 03 & 9,4 & 03 \\
É muito difícil agendar o exame & 00 & 0,0 & 02 \\
Não tem conhecimento de qual foi o resultado & 01 & 3,1 & 6,6 \\
Não gosta do profissional que o realiza & 00 & 0,0 & 01 \\
Outros motivos & 04 & 12,5 & 01 \\
\hline
\end{tabular}

Fonte: Dados coletados, ESF Delfino Magalhães e Jardim Palmeiras I e II, 2006.

*Foram escolhidas mais de uma opção

\section{SCUSSÃO}

Considerando os resultados desse estudo, pôde-se observar que a não realização do ECCU, na periodicidade correta, ou seja, nos últimos três anos ${ }^{(3)}$, na região pesquisada é baixa, abrangendo $1,5 \%$ das mulheres pesquisadas. Tal fato mostra boa cobertura do exame na área pesquisada, ultrapassando a meta estabelecida pela Organização Mundial de Saúde de, no mínimo, $80 \%$ de cobertura $^{(4,16)}$. Essa freqüência tem a limitação de ter sido feito em apenas um setor da cidade. Em estudo realizado em São Luís (MA), em mulheres na faixa etária de 25 a 49 anos, a freqüência de não realização do exame foi de $17,6 \%{ }^{(17)}$. Em Londrina (PR), essa freqüência foi de 19,3\%, entre as mulheres de 20 a $59 \operatorname{anos}^{(4)}$. Na Argentina, entre as mulheres de 18 a 64 anos, $69,5 \%$ não realizaram esse exame nos últimos três anos ${ }^{(10)}$.

Verificou-se que a idade pode ser um fator associado para a não realização do exame ECCU, sendo tal fato mais comum entre as mulheres com idade a partir de 50 anos, o que condiz com o observado nos estudos realizados em Londrina $(P R)^{(4)}$ e São Paulo $(S P)^{(14)}$. Ressalta-se que, essas mulheres nessa faixa etária podem estar sob maior risco, já que a incidência do CCU é maior nesta faixa etária ${ }^{(9)}$, sendo importante que as ações de prevenção contra este tipo de câncer estejam voltadas para essas mulheres, uma vez que neste estudo $4,1 \%$ das mulheres com 50 anos ou mais não realizam o exame, apesar de sua alta cobertura, sendo esse número aproximadamente três vezes maior em relação às mulheres mais jovens.

Assim como observado em estudos realizados em São Luís $(M A)^{(17)}$, São Paulo $(S P)^{(14)}$ e Rio Grande (RS) ${ }^{(13)}$, verificou-se que a baixa escolaridade é comum entre as mulheres que não se submetem ao ECCU, sendo esta, uma característica bastante identificada entre as mulheres com $\mathrm{CCU}^{(9,17,18)}$. Essa baixa escolaridade observada pode está associada ao fato da maior parte das pesquisadas terem 50 anos de idade ou mais, possuindo baixa escolaridade, ou seja, menos de oito anos de estudo. Já que, neste estudo foi observado que a baixa escolaridade está associada à não realização do ECCU pelas mulheres acima de 50 anos de idade. Tal fato pode está relacionado ao sistema educacional das décadas passadas, que possuía grandes deficiências e o acesso era restrito para as mulheres, que deveriam ser instruídas apenas para os serviços domésticos ${ }^{(19)}$.

Observou-se ainda, que a menor freqüência de realização deste exame também é comum entre as mulheres de baixa renda familiar $^{(13,17)}$ e entre as que não trabalham, já que, como visto neste estudo, o fato de não trabalharem está significativamente associado à não realização do ECCU pelas mulheres com faixa etária acima de 50 anos. Pode está relacionado a isto, à menor escolaridade e 
Rodrigues Neto JF, Figueiredo MFS, Siqueira LG. Exame citopatológico do colo do útero: fatores associados a não realização em ESF. Rev. Eletr. Enf. [Internet]. 2008;10(3):610-21. Available from: http://www.fen.ufg. br/revista/v10/n3/v10n3a07.htm

maior idade dessas mulheres, uma vez que o mercado de trabalho tem se mostrado bastante exigente quanto à qualificação profissional e optado por profissionais mais jovens, o que faz com que as mulheres que trabalham exclusivamente em casa tenham menor acesso às informações e menos autonomia para tomar decisões relativas à sua saúde ${ }^{(4)}$.

O comportamento inadequado das mulheres em relação ao ECCU pode ainda está relacionado ao seu estado conjugal. Estudo realizado em São Luís (MA) ${ }^{(17)}$ destacou o fato de que não ter um companheiro pode está associado a maior risco de não realizar o ECCU. Já neste estudo, observou-se, que 19 (42,2\%) das entrevistadas que não realizam o exame são casadas ou possuem união consensual, o que pode estar relacionado a aspectos como submissão destas mulheres aos companheiros, o pouco contato com outras pessoas para tratar de assuntos de saúde ${ }^{(10)}$, bem como a falsa idéia de que as mulheres com união estável são possuidoras de certo grau de imunidade às doenças sexualmente transmissíveis (DST), o que não se constitui em verdade absoluta, por não ser o casamento, um obstáculo para a multiplicidade de parceiros ${ }^{(9)}$.

Quanto aos aspectos sexuais e reprodutivos pesquisados, notou-se que uma parte considerável das entrevistadas, 15 $(33,3 \%)$, já teve mais de um parceiro sexual, o que as torna mais predispostas às DST, como o papilomavirus humano - HPV, sendo este o principal fator associado com a ocorrência do $\mathrm{CCU}^{(4,9,18)}$. Destaca-se também que a maioria das entrevistadas afirmou não fazer uso de nenhum tipo de métodos contraceptivos, podendo este fato está relacionado a não submissão ao ECCU, já que este procedimento pode está vinculado às atividades de planejamento familiar ${ }^{(10,11)}$. A multiparidade observada neste estudo pode constituir fator de risco para a ocorrência do $\mathrm{CCU}^{(18)}$. Observou-se que a idade de início das atividades sexuais de 18 anos ou mais está associada à não realização do ECCU pelas mulheres com idade de 50 anos ou mais, o que pode está relacionado ao fato das mulheres pesquisadas com idade mais avançada associarem esta característica ao menor risco de possuir esse tipo de câncer. A maior realização do exame nas mulheres jovens, na fase reprodutiva(4,10-11), pode está vinculada a procedimentos de rotina durante o pré-natal ou como parte do planejamento familiar ou ainda devido a recomendações médicas e queixas ginecológicas ${ }^{(7,10,12,14)}$ e não somente para prevenção do CCU.

Entretanto, deve-se considerar que o fato da grande parte das mulheres que não realizam o ECCU terem a idade acima de 50 anos, pode está associado à suas características socioeconômicas, culturais, sexuais e reprodutivas e não propriamente a idade, que pode ser um reflexo do tipo de sociedade em que viviam nas décadas de 50 e 60, que é diferente dos tempos atuais, já que atualmente, se tem maior facilidade de acesso à educação e ensino profissionalizante, as mulheres não são educadas apenas para serem "donas de casa", há maior liberdade sexual e alguns "tabus" quanto à sexualidade feminina já foram quebrados, além do maior acesso aos serviços de saúde. Acredita-se que as mulheres, dessas últimas décadas, não terão a variável idade como fator associado à não realização do exame citopatológico ${ }^{(19)}$.

As Políticas do Sistema de Saúde no Brasil, com o Programa de Atenção Integral a Saúde da Mulher, a partir do início da década de 80 , que introduziu a prática do ECCU no ato do atendimento ambulatorial ${ }^{(9)}$, bem como o Viva Mulher - Programa Nacional de Controle de Câncer de Colo de Útero e de Mama, em 1997, que pretendia estabelecer o diagnóstico precoce e tratamento adequado por meio da oferta de serviços através de uma rede nacional integrada(3), tem atingido bons resultados, principalmente em mulheres mais jovens. Contudo, destaca-se aqui, a importância da realização de campanhas e ações de combate ao CCU destinadas às mulheres de idade mais avançada e menor nível socioeconômico e cultural, a fim de eliminar especificamente os entraves para a compreensão da importância de realização do ECCU. Além disso, as equipes de ESF devem priorizar a realização deste exame a estas mulheres, independente da meta mensal a ser atingida.

O conhecimento das mulheres sobre a existência do ECCU, foi observado entre as 
Rodrigues Neto JF, Figueiredo MFS, Siqueira LG. Exame citopatológico do colo do útero: fatores associados a não realização em ESF. Rev. Eletr. Enf. [Internet]. 2008;10(3):610-21. Available from: http://www.fen.ufg. br/revista/v10/n3/v10n3a07.htm

entrevistadas, entretanto, parte considerável desconhecia a sua finalidade. A associação correta do ECCU com a prevenção ou detecção precoce do CCU foi feita por $17(37,7 \%)$ das pesquisadas, apresentando resultado semelhante ao encontrado nos estudos realizados no Brasil, em Natal (RG) ${ }^{(12)}$ e na Argentina, em Puerto Leoni ${ }^{(10)}$. É importante considerar que as mulheres que responderam incorretamente, que o ECCU era para detectar DST ou doenças ginecológicas; alterações no útero; ou outras doenças, pode está relacionado ao fato deste procedimento ocorrer durante a consulta ginecológica, onde esses outros aspectos também são considerados.

Verificou-se que o conhecimento sobre a finalidade do ECCU esteve associado à cor não branca, a ter o conhecimento correto sobre o exame, viver sem um companheiro e a trabalhar. Acredita-se que a associação entre a cor e o conhecimento sobre a finalidade do exame pode está relacionado a outras características socioeconômicas ou culturais e não propriamente a cor. Ressalta-se ainda, que apesar de muitas mulheres terem conhecimento sobre a finalidade do exame, há grande inconsistência entre o mesmo e a prática, já que não incorporam os conhecimentos e atitudes favoráveis sobre a prevenção no cotidiano do cuidado à própria saúde ${ }^{(6)}$.

Foi verificado que o principal motivo para a não realização do ECCU, referido pelas pesquisadas, foi o fato de não estarem doentes ou não apresentarem nenhuma sintomatologia, seguido pelo fato de terem vergonha ou se sentirem constrangidas, sendo estas justificativas, também, as principais relatadas nos estudos realizados em São Paulo (SP) ${ }^{(14)}$, Natal (RG) $)^{(12)}$ e Londrina (PR) ${ }^{(4)}$. Tal atitude das mulheres de não realizarem o exame devido à ausência de queixas ginecológicas, trata-se de uma construção cultural de significação dos fenômenos de saúde e doença, fundamentada no modelo instaurado historicamente pela medicina curativa, encorajando os indivíduos a buscarem cuidado médico, quando os sintomas reconhecíveis estão presentes ${ }^{(14)}$, o que foi bastante evidenciado principalmente entre as mulheres de 50 anos ou mais, já que 65,6\% dessas mulheres referiram este motivo. Trata- se também de um indicador de desconhecimento das ações preventivas por parte dessas mulheres, por acharem que para a realização do exame é preciso estar doente ${ }^{(10)}$. Desta forma pode-se explicar em parte, porque a maioria dos casos do CCU, que são diagnosticados pelo exame citopatológico já se apresenta numa fase tardia da doença, cujo prognóstico já não é tão alentador, contribuindo para a permanência das altas taxas de mortalidade por CCU, já que as mulheres, geralmente, reconhecem os sintomas característicos de uma fase mais tardia da doença ${ }^{(14)}$.

Em relação à vergonha, como motivo para não realização do ECCU, referida pelas mulheres desse estudo, tal sentimento pode está relacionado com a impessoalidade do procedimento; ao fato de ser tão invasivo; com a exposição do corpo; com a questão da sexualidade e dos "tabus" relacionados a este tema; e ainda sensação de impotência e perda do domínio e autonomia sobre o próprio corpo $^{(8,11-12,20)}$. Destaca-se que esse sentimento de vergonha exacerbado dificulta a realização do exame, já que a mulher não consegue relaxar e isto pode fazer com que esse exame se torne mais doloroso(6), levando assim, a outra questão a ser considerada, como o fato do exame citopatológico ser visto por muitas mulheres como uma experiência dolorosa ${ }^{(14)}$.

Deve-se considerar, ainda, que a não realização do exame também pode está associada a fatores relacionados com o sistema de saúde e seus profissionais ${ }^{(4,11,14)}$. O acesso inadequado ao atendimento básico pode desestimular as mulheres a procurarem os serviços de saúde, além de gerarem sentimentos de descontentamento e a indignação pela demora no atendimento ou agendamento do exame; pela falta de material para realização do mesmo e pelo número insuficiente de vagas ${ }^{(20)}$. Tal fato foi observado no estudo realizado em Saõ Luís $(M A)^{(17)}$, uma vez que os maiores percentuais para a não realização foram observados em mulheres que não realizaram consulta médica no último ano ou tiveram menor contato com os serviços de saúde. Em contrapartida, foi observado neste estudo que $43(95,5 \%)$ das mulheres que não 
Rodrigues Neto JF, Figueiredo MFS, Siqueira LG. Exame citopatológico do colo do útero: fatores associados a não realização em ESF. Rev. Eletr. Enf. [Internet]. 2008;10(3):610-21. Available from: http://www.fen.ufg.br/revista/v10/n3/v10n3a07.htm

realizavam o ECCU utilizaram o serviço de saúde no último ano, tendo sido, estas, oportunidades para a realização do exame.

A atuação das equipes de saúde da família na região pesquisada pode ter tido influência na boa cobertura do ECCU. No entanto, são necessárias ações que visem atingir a população sob maior risco e de menor cobertura, já que a maior parte dos exames preventivos são realizadas nas mulheres com menor risco, apresentando menor impacto epidemiológico ${ }^{4-}$ 5)

O êxito no rastreamento CCU para redução do seu quadro de morbimortalidade depende, acima de tudo, da ação governamental para reorganização da assistência clínico-ginecológica às mulheres nos serviços de saúde, como foi visto neste estudo com a implantação da ESF. Faz-se necessário ainda, garantir a equidade do acesso ao serviço e a realização de campanhas específicas e de educação em saúde para mulheres de maior risco; a capacitação dos profissionais de saúde, em especial dos agentes comunitários de saúde que são o elo entre a comunidade e a Estratégia Saúde da Família( ${ }^{(4,9-10,14)}$.

\section{CONCLUSÕES}

De acordo com os objetivos estabelecidos para a realização do presente estudo verificouse que as mulheres que não realizaram o ECCU tiveram como principais fatores a baixa escolaridade; idade mais avançada; renda familiar menor que $\mathrm{R} \$ 600,00$ e não trabalhar fora de casa. Atualmente, as mulheres assumiram novos papéis na sociedade, contribuindo para o auto-conhecimento da sua saúde. Concomitantemente, aconteceram mudanças no sistema público de saúde brasileiro, aumentando o acesso aos serviços de saúde para as mulheres. As mulheres mais jovens, com maior escolaridade e nível econômico e ainda que trabalhem fora de casa, em relação a prevenção do câncer do colo do útero, tem utilizado mais esse serviço, especialmente no Programa Saúde da Família, que foi objeto desse estudo.

Neste trabalho também foi identificado que as mulheres com 50 anos de idade ou mais, que não realizavam o ECCU, tiveram com maior frequência o iníco da atividade sexual com 18 anos ou mais. Os principais motivos para a não realização do ECCU foram o fato de não estarem doentes ou não apresentarem nenhuma sintomatologia, seguido pelo fato de terem vergonha ou sentirem constrangidas.

As mulheres com as variáveis: cor não branca, conceituação correta sobre a finalidade do ECCU, viver sem companheiro e trabalhar fora de casa foram as que tiveram melhor conhecimento sobre a finalidade do ECCU.

Concluindo, pode-se dizer que a ESF, neste estudo, teve boa cobertura quanto à realização do ECCU, entretanto esforços precisam ser feitos para alcançar as mulheres com maior risco, desenvolvendo a atitude da procura pelo serviço de saúde para a realização de exames de prevenção, independente da presença de sintomas.

\section{REFERÊNCI AS}

1. International Agencyfor Reserch on Câncer [Internet]. Lyon (FR): IARC; c2008. [cited 2008 set 30]. Available from: http://www.iarc.fr/.

2. Chubaci RYS, Merighi MAB. Exame para a detecção precoce do câncer cérvico-uterino: vivência das mulheres da cidade de Kobe e Kawasaki, Japão e São Paulo, Brasil. Revista Brasileira Saúde Materno Infantil [Internet] 2005 [cited 2008 set 30]; 5(4):471-81. Available

from: http://artigocientifico.uol.com. br/uploads/artc 1140706351_13.pdf.

3. Ministério da Saúde; Instituto Nacional do Câncer INCA. Câncer de colo de útero. [Internet]. Rio de Janeiro: Ministério da Saúde; c1996-2008 [cited 2008 set 30]. Available from: http://www. inca.gov. br/conteudo_view. asp?id=326.

4. Silva DW, Andrade SM, Soares MA, Turini B, Schneck CA, Lopes MLS. Cobertura e fatores associados com a realização do exame Papanicolaou em município do Sul do Brasil. Revista Brasileira de Ginecologia e Obstetrícia [Internet]. 2006 [cited 2008 set 30];28(1):2431. Availabe from: URL: http://www. scielo.br/pdf/rbgo/v28n1/29590.pdf 5. Pinho AA, França-Junior I. Prevenção do câncer de colo do útero: um modelo teórico para analisar o acesso e a utilização do teste de Papanicolaou. Revista Brasileira de Saúde Materno Infantil [Internet]. 2005 [cited 2008 
Rodrigues Neto JF, Figueiredo MFS, Siqueira LG. Exame citopatológico do colo do útero: fatores associados a não realização em ESF. Rev. Eletr. Enf. [Internet]. 2008;10(3):610-21. Available from: http://www.fen.ufg.br/revista/v10/n3/v10n3a07.htm

set 30];3(1):95-112. Availabe from: http://www. scielo.br/pdf/rbsmi/v3n1/a12v03n1.pdf.

6. Ferreira MLM; Oliveira C. Conhecimento e significado para funcionárias de indústrias têxteis sobre prevenção do câncer do colouterino e detecção precoce do câncer da mama. Revista Brasileira de Cancerologia [Internet]. 2006 [cited 2008 set 30];52(1):5-15. Available from:

http://www.inca.gov.br/rbc/n_52/v01/pdf/artigo1.pdf 7. Motta EV, Fonseca AM, Bagnoli VR, Ramos LO, Pinotti JA. Colpocitologia em Ambulatório de Ginecologia Preventiva. Revista da Associação Médica Brasileira [Internet]. 2001 [cited 2008 set 30];47(4):302-10. Available from: http://www.scielo.br/pdf/ramb/v47n4/7396.pdf.

8. Paula AF, Madeira AMF. O exame colpocitológico sob a ótica da mulher que o vivencia. Revista Escola de Enfermagem USP [Internet]. 2003 [cited 2008 set 30];37(3):8896. Available from: http://www. ee.usp. br/reeusp/upload/pdf/173. pdf.

9. Guedes TG, Pordeus AMJ, Diógenes MAR. Análise epidemiológica do câncer de colo de útero em serviço de atendimento terciário no Ceará - Brasil. Revista Brasileira em Promoção da Saúde [Internet]. 2005 [cited 2008 set 30]; 18(4):205-10. Available from: http://redalyc.uaemex. mx/redalyc/pdf/408/408 18408.pdf.

10. Gamarra CJ, Paz EPA, Griep RH. Conhecimentos, atitudes, prática do exame de Papanicolaou entre as mulheres argentinas. Revista Saúde Pública Rio de Janeiro [Internet] 2005 [cited 2008 set 30];39(2):270-6. Available from: http://www.scielo.br/pdf/rsp/v39n2/24052.pdf. 11. Pelloso SM, Carvalho MDB, Higarashi IH. Conhecimento das mulheres sobre o câncer cérvico-uterino. Acta Scientiarum. Health Sciences [Internet] 2004 [cited 2008 set 30]; 26(2):319-24. Availabe from: http://www. periodicos. uem.br/ojs/index.php/Ac taSciHealthSci/article/viewFile/1582/935.

12. Davim RMB, Torres GV, Silva RAR, Silva, DAR. Conhecimento de mulheres de uma Unidade Básica de Saúde da cidade de Natal/RN sobre o exame de Papanicolau. Revista Escola de Enfermagem USP [Internet]. 2005 [cited 2008 set 30]; 39(3):296-302. Available from: http://www.ee.usp. br/reeusp/upload/pdf/10.pdf 13. Cesar JA, Horta BL, Gomes G, Houlthausen RS, Willrich RM ,Koercher $A$, et al. Fatores associados à não realização de exame citopatológico de colo uterino no extremo Sul do Brasil. Cadernos de Saúde Pública [Internet]. 2003 [cited 2008 set 30]; 19(5):1365-72. Available

from: http://www. scielo.br/pdf/csp/v19n5/17808.pdf. 14. Pinho AA, França Junior I, Schraiber LB, Oliveira AFPL. Cobertura e motivos para a realização ou não do teste de Papanicolaou no Município de São Paulo. Cadernos de Saúde Pública [Internet]. 2003 [cited 2008 set 30]; 19 Suppl 2:S303-13. Availabe from: http://www. scielo.br/pdf/csp/v19s2/a12v19s2.pdf.

15. Ministério da Saúde. Secretaria de Atenção à Saúde. Departamento de Atenção Básica. SIAB: Manual do Sistema de Atenção Básica. 1a edição. Brasília (Brasil): Ministério da Saúde; 2003.

16. World Health Organization. Cervical cancer screening in developing countries: report of a WHO consultation. Geneva (Switzerland): World Health Organization; 2002.

17. Oliveira MMH, Silva AAM, Brito LMO, Coimbra LC. Cobertura e fatores associados à não realização do exame preventivo de Papanicolaou em São Luís, Maranhão. Rev. Bras. Epidemol. [Internet]. 2006 [cited 2008 set 30];9(3):325-34. Availabe from: http://www. scielo. br/pdf/rbepid/v9n3/06.pdf. 18. Lima CA, Palmeira JAV, Cipolotti R. Fatores associados ao câncer de colo uterino em Própria, Sergipe, Brasil. Cad. Saúde Pública [Internet]. 2006 [cited 2008 set 30]; 22(10):2151-56. Availabe from: http://www. scielo.br/pdf/csp/v22n10/14.pdf.

19. Biasoli-Alves ZMM. Continuidades e rupturas no papel da mulher brasileira no século $X X$. Psicologia: Teoria e Pesquisa [Internet]. 2000 [cited 2008 set 30];16(3):233-9. Availabe from:

http://www.scielo.br/pdf/ptp/v16n3/4810.pdf.

20. Duavy LM, Batista FLR, Bessa MS, Santos JBF. A percepção da mulher sobre o exame preventivo do câncer cérvico-uterino: estudo de caso. Ciência e Saúde Coletiva [Internet]. 2007 [cited 2008 set 30];12(3):733-42. Availabe from:

http://www. scielo.br/pdf/csc/v12n3/24.pdf.

Artigo recebido em 25.09.07

Aprovado para publicação em 30.09.08 